\title{
New method for characterizing the state of optical and opto-mechanical systems
}

\author{
Ritva Keski-Kuha, Babak Saif, Lee Feinberg \\ NASA Goddard Space Flight Center, Greenbelt Maryland 20771 \\ David Chaney \\ Ball Aerospace \& Technology Corp, Boulder, CO, USA 80301 \\ Marcel Bluth \\ ATK Space Systems, Magna, Utah 84044 \\ Perry Greenfield, Warren Hack, \\ Space Telescope Science Institute ,3700 San Martin Drive, Baltimore, MD 21218. \\ Scott Smith \\ NASA Marshall Space Flight Center, Huntsville, Alabama \\ James Sanders \\ GSFC/Vantage Systems Inc. 4600 Forbes Blvd, Lanham, MD 20706
}

\begin{abstract}
James Webb Space Telescope Optical Telescope Element (OTE) is a three mirror anastigmat consisting of a $6.5 \mathrm{~m}$ primary mirror (PM), secondary mirror (SM) and a tertiary mirror. The primary mirror is made out of 18 segments. The telescope and instruments will be assembled at Goddard Space Flight Center (GSFC) to make it the Optical Telescope Element-Integrated Science Instrument Module (OTIS). The OTIS will go through environmental testing at GSFC before being transported to Johnson Space Center for testing at cryogenic temperature. The objective of the primary mirror Center of Curvature test $(\mathrm{CoC})$ is to characterize the PM before and after the environmental testing for workmanship. This paper discusses the $\mathrm{CoC}$ test including both a surface figure test and a new method for characterizing the state of the primary mirror using high speed dynamics interferometry.
\end{abstract}

Key words: James Webb Space Telescope, JWST, vibration, optical measurement

\section{INTRODUCTION}

James Webb Space Telescope (JWST) is scheduled to be launched in 2018. The JWST telescope is a three mirror anastigmat comprised of a segmented primary mirror, a convex secondary mirror, and a tertiary mirror. Additionally there is a flat fine steering mirror used to maintain alignment of the telescope to the instrument module. Figure 1 shows an artist rendering of the completed telescope. In order to fit within the rocket fairing the entire assembly will be folded up and then deployed on orbit. For this reason the primary mirror is segmented consisting of 18 hexagonal mirrors each approximately 1.5 meters point to point. The mirror segments are constructed from a lightweight beryllium substrate with both a radius-of-curvature actuation system and a six degree-of-freedom hexapod actuation system. Once completed the eighteen PMSAs are mounted to the primary mirror backplane support structure, a graphite/epoxy composite truss structure. ${ }^{4}$ All mirrors are coated with protected gold. 


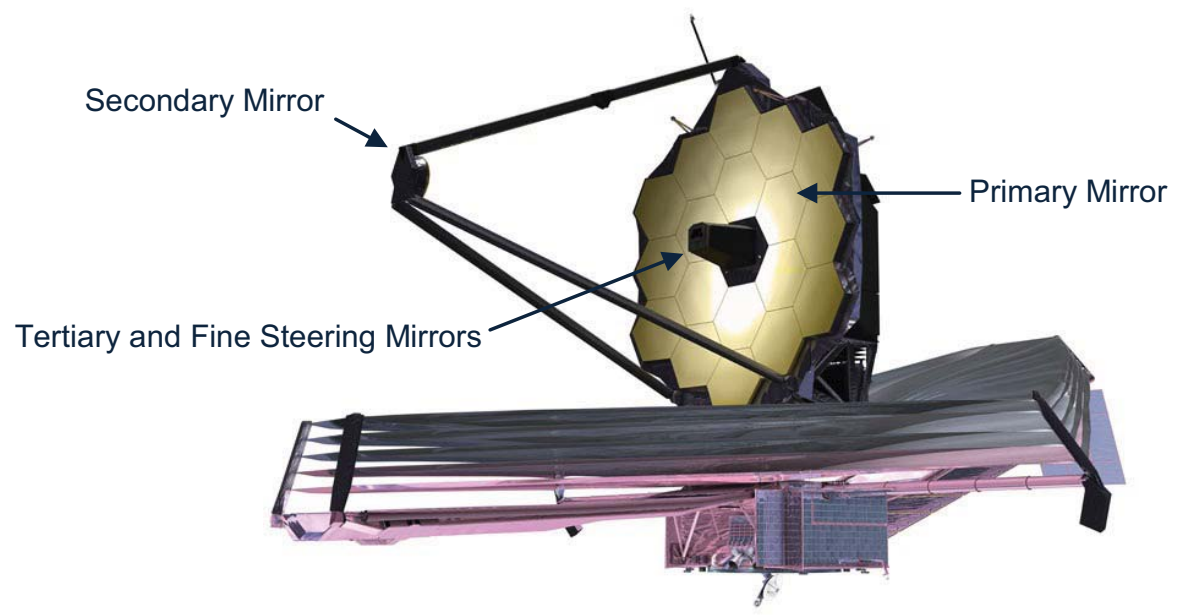

Figure 1: Artist rendering of JWST telescope

The telescope will be assembled and instruments installed at NASA Goddard Space Flight Center clean room facility. The Integrated Science Instrument Module (ISIM) consists of four science instruments in its instrument housing which is attached to the backplane of the Primary mirror. The primary mirror will be measured before and after environmental exposure by measuring, primary segments 'surfaces' to gain insight into the state of the assembled telescope. This includes Power Spectral Densities (PSD), spatial modes, phase and modulation transfer functions of the full telescope assembly. State of the telescope assembly will be measured before and after environmental exposure using a high speed interferometer. This interferometer has been designed and built in collaboration with 4D Technology ${ }^{5}$. Environmental exposure simulates the launch conditions for the telescope assembly.

\section{TEST OVERVIEW}

The primary mirror segments will be tested statically and dynamically by measuring the surface of each segment using a Computer Generated Hologram $(\mathrm{CGH})$ at Center of Curvature $(\mathrm{CoC})$ of the Primary mirror. Primary instrument to perform static and dynamics measurements is a high speed interferometer designed and fabricated by 4D technology. It is equipped with a high power $25 \mathrm{~mW}$ He-Ne laser with path matching capability to keep the integration times short. The interferometer captures all of the phase shifted interferograms in a single camera frame. The heart of the system is a pixel-wise phase-shifting element in which each pixel has a unique phase-shift. The camera is a high-speed CMOS camera that is capable of capturing frames at a rate greater than $2 \mathrm{KHz}$. The frame rate is a function of the size of the region of interest selected for data transfer. Interferometer can take data at $1 \mathrm{KHz}$ for $720 \times 720$ pixel formats. Higher speed of $2.25 \mathrm{KHz}$ can be achieved at $400 \times 400$ pixels formats. This interferometer allows rigid body measurements to get the modes and change in the mode shapes and frequencies. It also allows deformation measurements such as astigmatism at $250 \mathrm{~Hz}$ and deformation due to inertia of the mirror to rigid body motions.

The static surface measurements of the segments will measure changes to the surfaces before and after environmental vibration and acoustics tests. Predicated change to astigmatism of the segment is about $10 \mathrm{~nm}$ RMS. The static surface measurements are fit to Zernike's before and after environmental test. Repeatability of static measurements in the clean room sets the noise level for this test. Repeatability of the static measurements is a strong function of temperature and its gradients. Test on JWST flight spares has been done to measure the noise level in the clean room for the static measurements. The surface RMS noise floor is $10 \mathrm{~nm}$ and astigmatism RMS noise level is $20 \mathrm{~nm}$. This is achieved under tight temperature control in the clean room.

The dynamics test is done by stimulating the telescope assembly at several places and measuring the dynamics behavior of the primary segments at $\mathrm{CoC}$ through a $\mathrm{CGH}$, just like the static case. The stimuli are applied at the hard points of the telescope assembly. Vibration levels are in micron and the frequencies are spread over $3 \mathrm{~Hz}$ to $250 \mathrm{~Hz}$. Both random and sine vibration are measured. PSDs, spatial modes, phase and modulation transfer functions are measured. PSDs per pixels are measured by temporal phase unwrapping individual pixels and FFT the displacement 
in time. This is also normalized to PSDs of the forcing function of the stimuli. Spatial modes are measured by creating surfaces by temporal phase unwrapping at each time. Surfaces are fitted to Zernike's. FFT each coefficient of Zernike's are normalized at each frequency to amplitude and phase of the force function. These normalized amplitudes and phases are compared before and after environmental test. Individual modes, rigid body and deformation modes are observed by temporal phase unwrapping of each pixel and observing all the pixels at the same time. These modes include absolute piston and also deformation at point of contacts between back structures and the mirror due to mirror inertia as they move as a rigid body. Forcing function of the stimuli is used as a reference to tie the segment measurement together as a measure of backplane structural modes. Although segments are measured one at the time using the phase and magnitude of the forcing functions as references enables observation of the backplane modes. Transfer functions are used to measure the changes to load path and stiffness of the backplane before and after environmental test.

\subsection{Metrology Validation}

The primary mirror segments are each off-axis sections of the parent conic. There are three prescription types defined as A, B, and C segment type. For the JWST telescope one spare primary mirror segment was manufactured for each of the three prescription types. The A segment spare is also called the EDU or engineering development unit, as it was the first JWST primary mirror segment built. This mirror has been through all processing including cryo-null figuring, a process where the mirror is polished for optimized cryogenic performance, and gold coating. The C segment spare has been polished but not cryo-null figured or coated. Finally, the B spare has not been polished and is in a rough ground state. In order to develop and validate the metrology described in this paper the A segment spare or the EDU was used. Further testing will be done using $\mathrm{C}$ spare mirror. The EDU was mounted on a test stand within the Spacecraft Systems Development and Integration Facility (SSDIF) cleanroom at NSA/Goddard Space Flight Center. This test stand was originally used at SSG-Tinsley for ambient optical metrology during the polishing process. Figure 2 shows a picture of the EDU and C7 mirrors on the test stand.
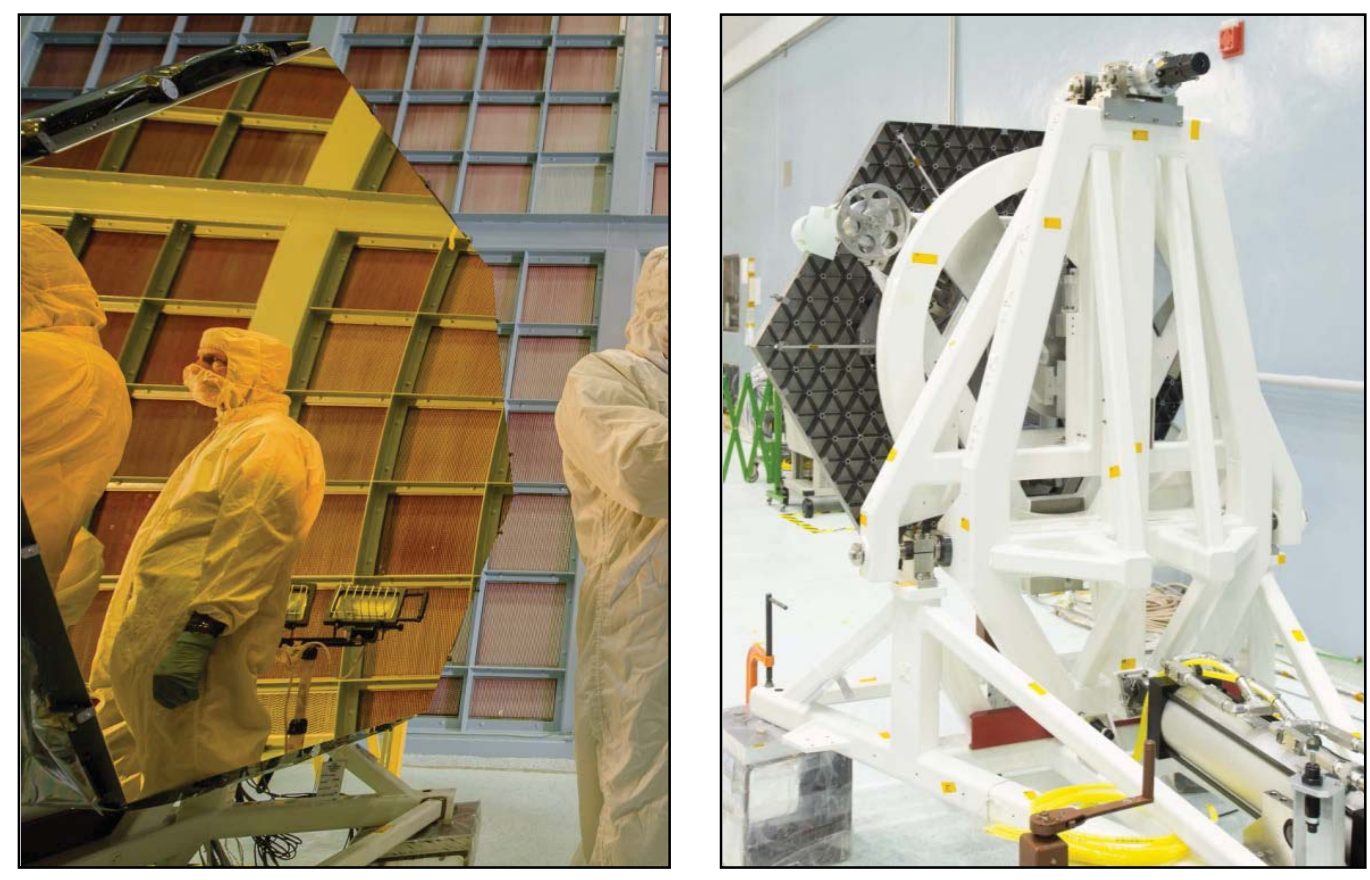

Figure 2: EDU \& C7 mirrors on test stand.

The optical test layout employed for primary mirror testing is that of a classical interferometric center of curvature test. This test involves the placement of an interferometer with a diverging lens such that its focus is coincident with the center of curvature of the mirror under test. If the test mirror were a sphere then all rays would be normal to the 
mirror surface. However, as previously described the JWST primary mirror segments are not spheres but rather offaxis aspheres. Therefore, a CGH (computer generated hologram) is required to convert the spherical test beam to an aspheric one. When the mirror is placed at the correct location relative to the CGH then all test rays are normal to the mirror surface. Essentially the CGH is acting as a portion of a diffractive null lens allowing for full interferometer spatial resolution of an off-axis segment. Figure 3 shows a simplified layout of the test setup.

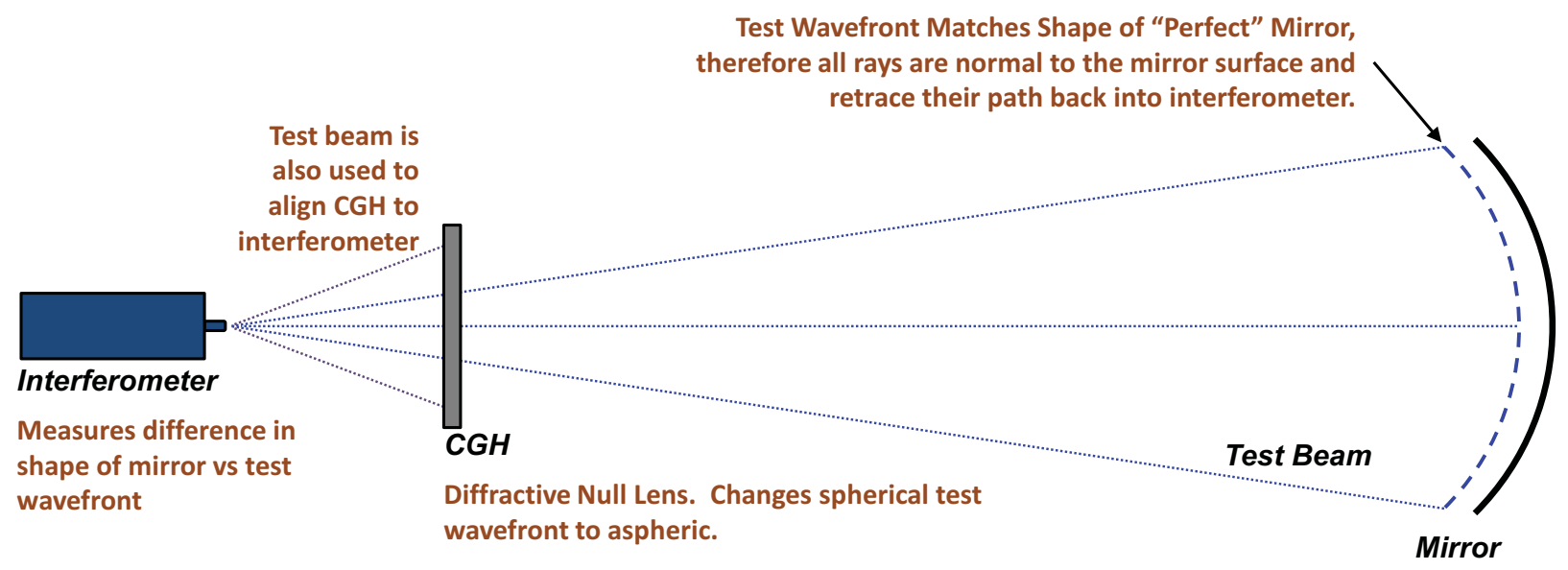

Figure 3: optical test layout

The six degree of freedom alignment of the CGH to the mirror segment is critical and is set using several different metrology devices. First the CGH is aligned to the interferometer using features built into the CGH design. Once set the CGH-to-interferometer alignment remains fixed and is moved as a unit. This is accomplished by mounting both pieces of optical equipment on a breadboard which itself rests on top of a six degree of freedom rotopod built by Mikrolar Inc. The rotopod provides for a good range of motion with a high load capacity. The stage was also designed to give micron and sub arcsecond level motion resolution.

The alignment of the CGH to the mirror segment is broken up into three components. The tip/tilt of the mirror is aligned using interferometer tilt fringes as feedback. The decenter and clocking of the mirror relative to the CGH is achieved using an alignment camera system. This system is mounted on the same breadboard as the interferometer and consists of a camera attached to a Meade telescope. The system relies on additional features built into the CGH diffractive pattern as well as fiducial targets mounted to the sides of the mirror. The final alignment degree of freedom is the axial spacing between the mirror and the CGH. This distance is measured using a Leica ADM (absolute distance meter) and set accordingly. Figure 4 shows a modeled representation of the optical metrology setup.

The radius of curvature of the primary mirror is approximately 16 meters. This is the distance that the mirror must be positioned away from the interferometer in a center of curvature test. A long optical path test such as this leads to a number of challenges, the most notable of which is turbulence and stratification within the air path. This was especially challenging for the EDU test as the optical setup was positioned right next to the cleanroom air outlet. However, we were able to work closely with the Spacecraft Systems Development and Integration Facility (SSDIF) facilities personnel to optimize the thermal environment. This was also very important as the primary mirror segments are designed for cryogenic use. During room temperature testing the thermal gradients within the mirror assembly cause very large and measureable changes to the figure, radius of curvature, and astigmatism contributions. In order to minimize these effects the temperature within SSDIF needed to be very stable. A rate of change requirement of less than $0.05 \mathrm{C}$ air temperature delta per 10 minutes was established as the limit. This keep mirror gradients, and thus mirror deformations, within acceptable values. Figure 3 shows the test layout and location within the cleanroom. 


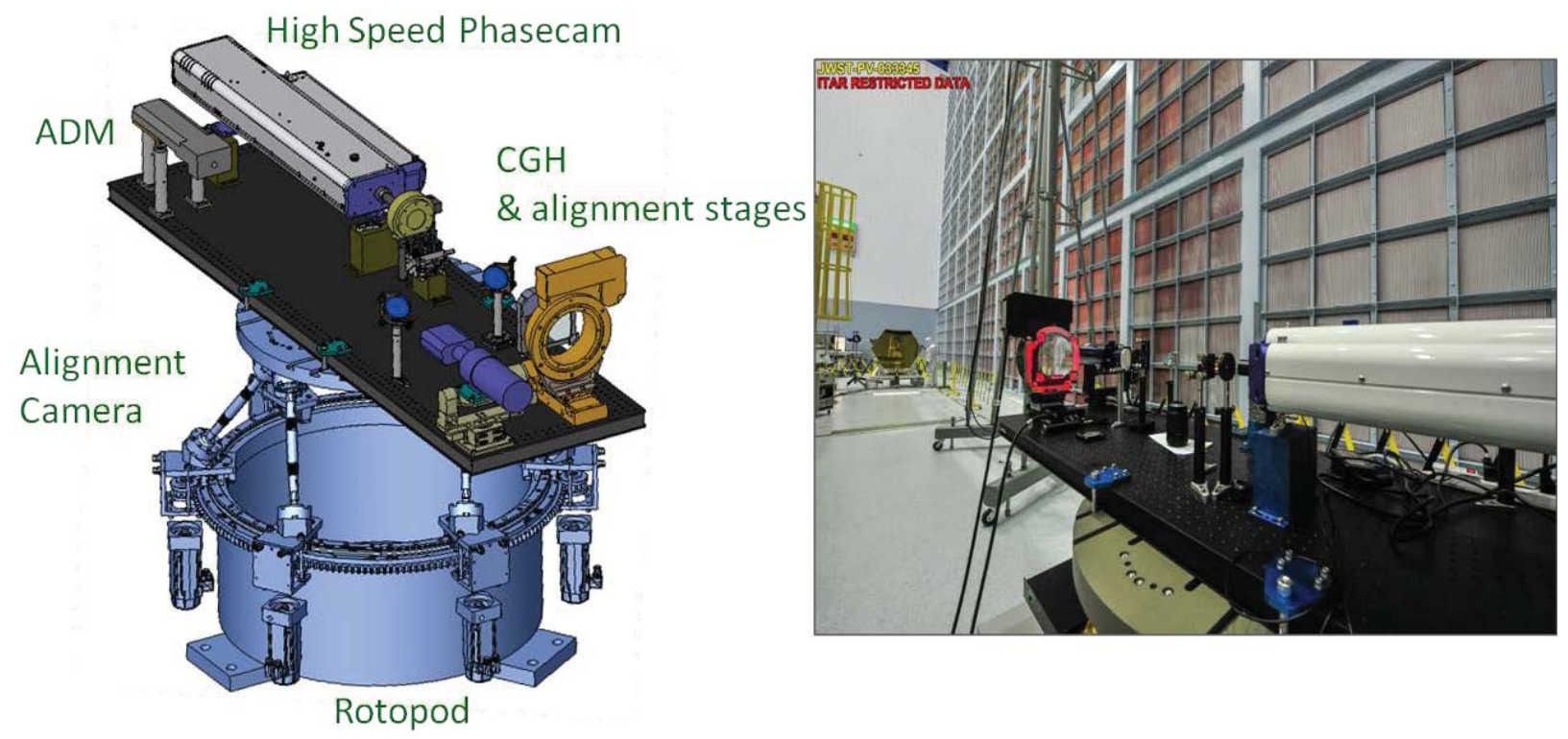

Figure: 4: Center of Curvature Test Layout for EDU Testing

\section{STATIC MEASUREMENT RESULTS}

Static surface figure measurements of each of the primary mirror segments will be used to look for any detrimental changes to them following the OTIS level vibro-acoustic testing. This is accomplished by performing a measurement both before and after vibration tests and then simply subtracting them from one another. This delta measurement philosophy is of key importance when establishing the metrology setup and requirements. Absolute errors, provided they are repeatable, will not affect the measurement capability. A discussion of all errors associated with the static measurement test is beyond the scope of this paper. However a quick summary of the errors that will potentially degrade the measurement results include: alignment of the interferometer to the CGH, alignment of the mirror to the $\mathrm{CGH}$, proper registration of the figure data, interferometer/diverger error, vibration effects, optical air path turbulence and stratification, primary mirror segment thermal gradients, primary mirror segment absolute soak temperature, and orientation changes relative to gravity. For each of the effects listed here the absolute error is not of concern but rather the difference between the before and after measurements. Therefore, the sum of all metrology errors will be called the measurement reproducibility. To get a sense of the level of this reproducibility error a series of measurements were taken with the EDU mirror. The testing took place over the course of four days and included four distinctive series of measurements. Each being an average of 250 fringe captures from the interferometer. To properly assess reproducibility the system was completely realigned for each measurement and the time of day and operator were varied.

The SSDIF thermal environment had not been optimized at the time of these measurements so monitoring of the air temperature was used to pick reasonable times to perform the measurements. Figure 5 shows the difference in each measurement from the other three. 


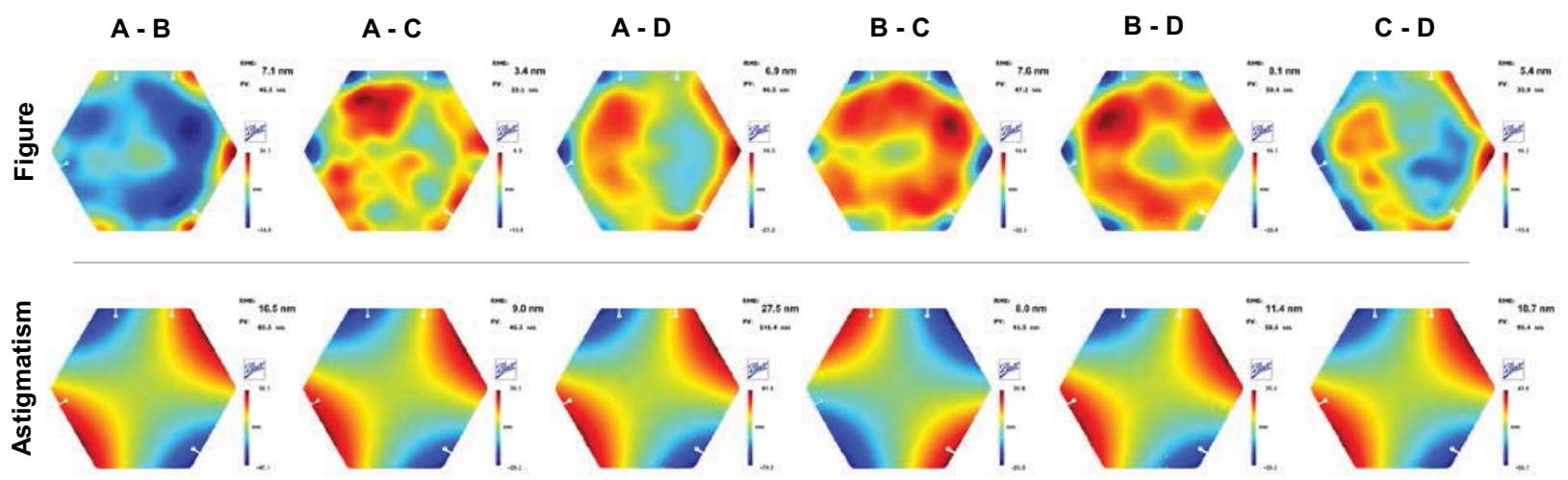

Figure 5: Results of EDU Reproducibility Testing

Reproducibility testing showed that metrology was good to $8.1 \mathrm{~nm}$ rms figure and $27.5 \mathrm{~nm}$ rms astigmatism worst case. The goals were $15 \mathrm{~nm}$ rms and $25 \mathrm{~nm}$ rms respectively. We will do further testing in the area where the OTIS level testing will occur in the cleanroom. We expect that the measured reproducibility numbers will improve due to better thermal control, especially in the case of the astigmatism.

In addition to reproducibility measurements the opportunity was taken to compare the EDU measurements performed in SSDIF to those taken previously at Marshall Space Flight Center's XRCF (X-Ray Cryogenic Facility). $\mathrm{XRCF}$ measurements were taken at ambient temperature but under vacuum in a cryogenic chamber. This comparison is used as a rough cross-check of previous results and also to give us confidence in the SSDIF results. Results shown in figure 6 confirm that SSDIF measurements closely match those taken at the XRCF. Results of this comparison are expected to be higher than a single facility reproducibility measurement since additional errors must be considered such as the use of two different CGHs, a different thermal environment, effects of the XRCF cryo chamber window, and XRCF vacuum chamber effects. While we back out these measurement perturbations to the level we know them, their uncertainties will add to the expected differences in the comparison. The measured figure delta of $12.6 \mathrm{~nm} \mathrm{rms}$ is consistent with the XRCF ambient metrology ${ }^{5,6}$ figure uncertainty of approximately $9 \mathrm{~nm}$ rms and the SSDIF uncertainty of closer to 10 to $12 \mathrm{~nm}$ rms. The astigmatism comparison, on the other hand, is a little higher than we would like. However, this is not completely unexpected given the likely thermal air effects on the SSDIF measurements due to the test setup position right next to the HEPA air outlet wall. To verify this is the cause of the astigmatism difference we will attempt to repeat this comparison once the EDU is moved out to the center of the SSDIF cleanroom.

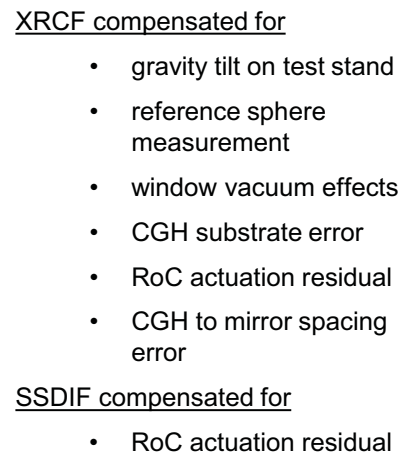

- RoC actuation residual

- $\mathrm{CGH}$ to mirror spacing error

$\underline{\text { SSDIF compensated for }}$

- RoC actuation residual

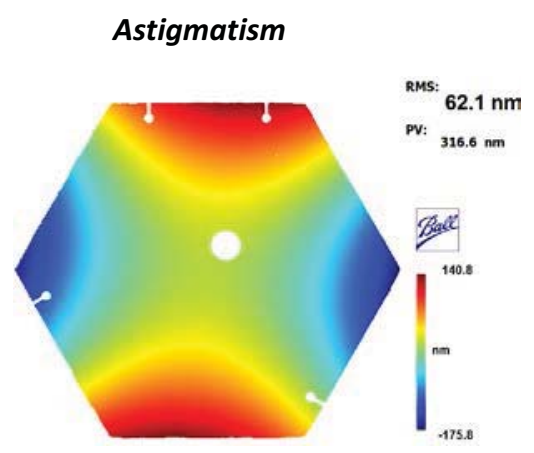

Figure

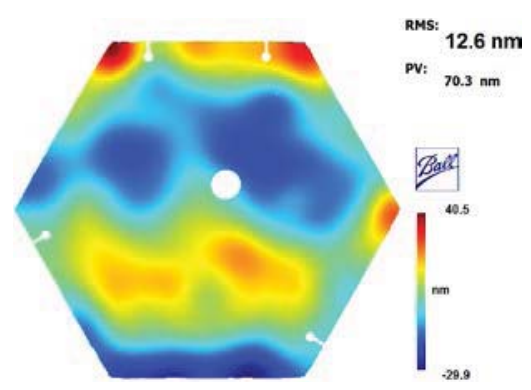

Figure 6: Difference between SSDIF and XRCF surface figure measurements. 


\section{DYNAMIC MEASUREMENT RESULTS}

The objective of the dynamics test was to determine if a methodology could be developed with sufficient sensitivity to detect changes in the mechanical configuration in the before and after states of the OTE as it goes through acoustic and vibration testing. Transfer functions represent a process of converting an input signal to an output observation. If the test article changes then the transfer function will change. Adding or losing mass, changes in stiffness due to structural changes and load path changes will change transfer functions. The goal of the EDU dynamics test was to demonstrate that the HSI can be used to characterize the full transfer function of the EDU Primary Mirror Segment Assembly (PMSA) surface for different stimulus conditions. The test stand (not the EDU) was altered to demonstrate the ability to measure changes in the transfer function as evidenced by shifts in the patterns of gain and phase lag over the surface of the PMSA. Gain and phase lag patterns over the surface of the PMSA are of particular interest for OTE structural integrity verification at CoC test. Shifts in gain and phase patterns are a mathematical window that looks behind the mirror segments and indicates that changes to the structure may exist. These can be used to "peer" into the OTE structure behind the mirrors. Figure 7 shows modulation and phase transfer function for a rigid body mode at $55 \mathrm{~Hz}$. Figure 8 shows residual deformation due to rigid body motion at $55 \mathrm{~Hz}$.- due to inertia of mirror's rigid body.
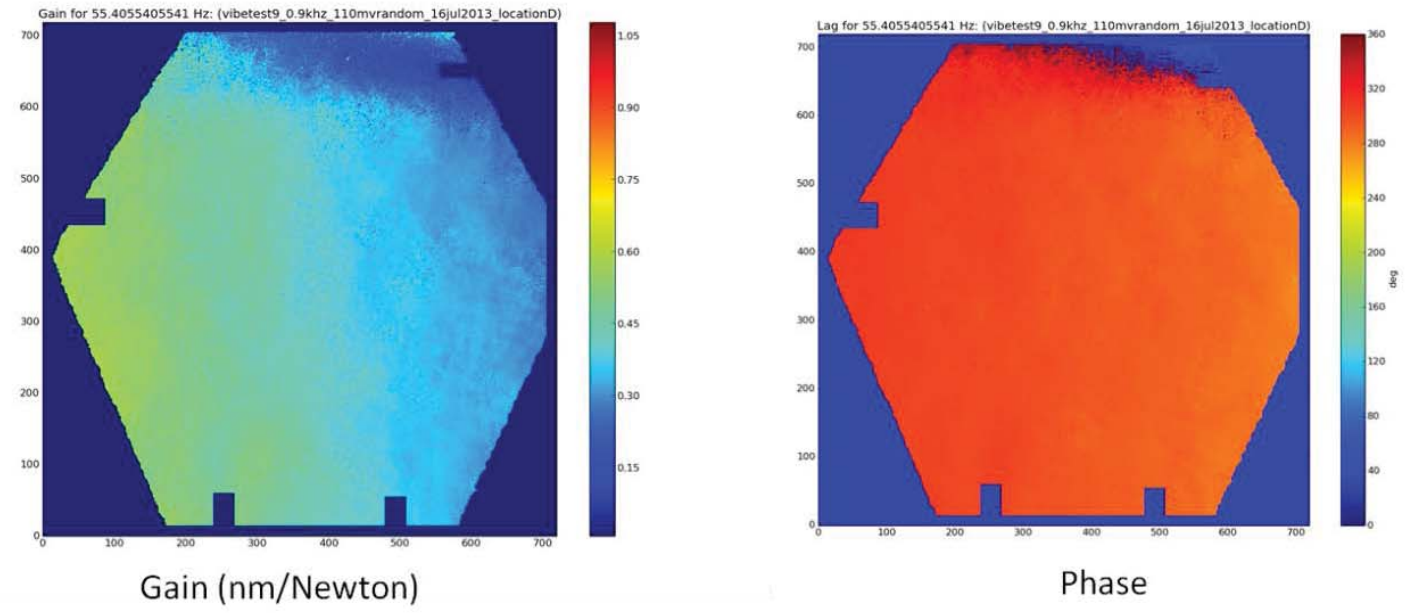

Figure 7. Modulation and Phase Transfer Function for a Rigid Body Mode at $55 \mathrm{~Hz}$
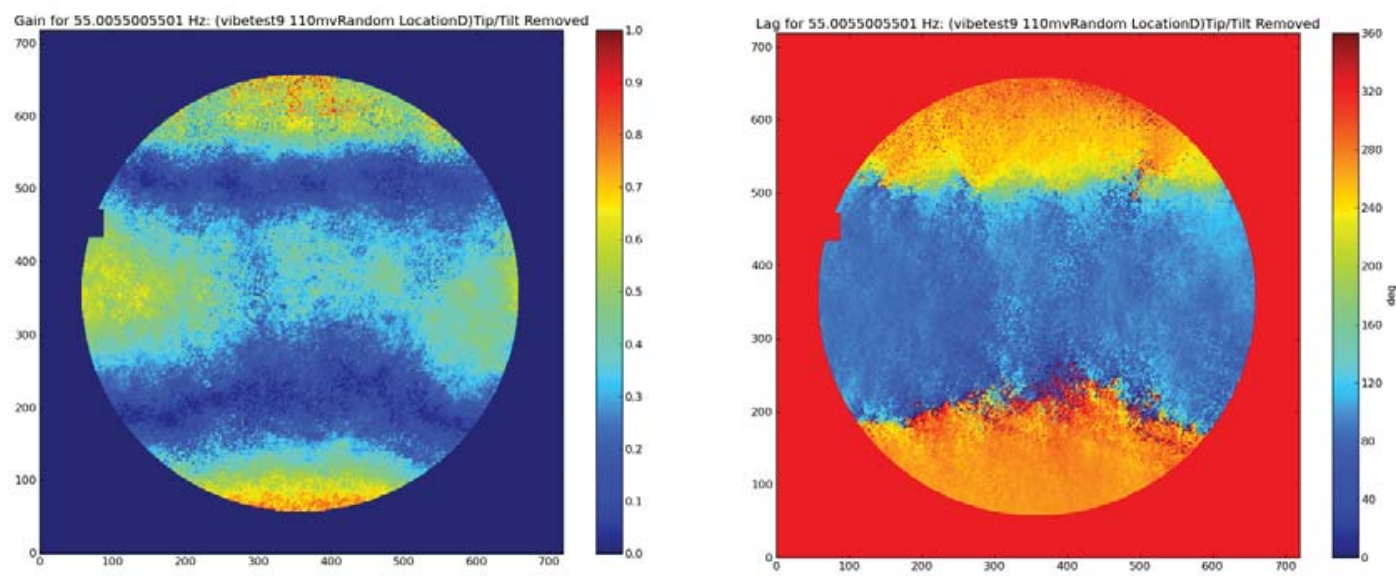

Figure 8. Residual Deformation due to Rigid Body Motion at $55 \mathrm{~Hz}$ - due to inertia of mirror's rigid body. 
In preparation for the test two modal surveys were conducted to characterize the mechanical dynamics of the bare test stand and the PMSA EDU mirror segment/test stand combination. The test configuration and model is shown in Figure 9a, with some of the accelerometer and impact locations shown. These tests provided useful information in identifying resonances as measured by the optical measurement system and in determining which resonances were largely mirror response and which were coupled responses of the mirror/stand combination. Data from these modal surveys was also used to refine the math models of these components and in evaluating structural changes induced in the test stand that simulate different before and after conditions that might occur from an acoustic test, for example. In fact, the primary purpose of these tests was to determine whether the combined mechanical and optical measurement system will be sufficiently sensitive to detect any changes in the OTIS condition before and after its acoustic test.

The control of the various test hardware items along with the data flow is represented in Figure 9b. Function generator sends a string of pulses that serves to trigger both the data acquisition system (DAQ) and the high speed interferometer (HSI), as well as control the frame rate of the HSI. The DAQ also sent an excitation signal to the shaker, which was connected to the mirror/test stand hardware via a stinger (a slender rod designed to be stiff along its axis but weak in the cross-axes and in bending), to provide excitation to the system under test. Various excitations were available and used; random, sine dwell, and sine sweep. The signal generator and DAQ were under control of a workstation, which also recorded the force and acceleration data from the DAQ. The images from the HSI were recorded on a separate workstation. Because a common trigger pulse was used for both the HSI and the DAQ, the mechanical data was correlated to the image data with a high degree of precision. An additional preparatory test was conducted to quantify the precision of simultaneity of the start of recording between the two systems, and they were found to start recording within $\pm 10 \mu \mathrm{sec}$ of each other.
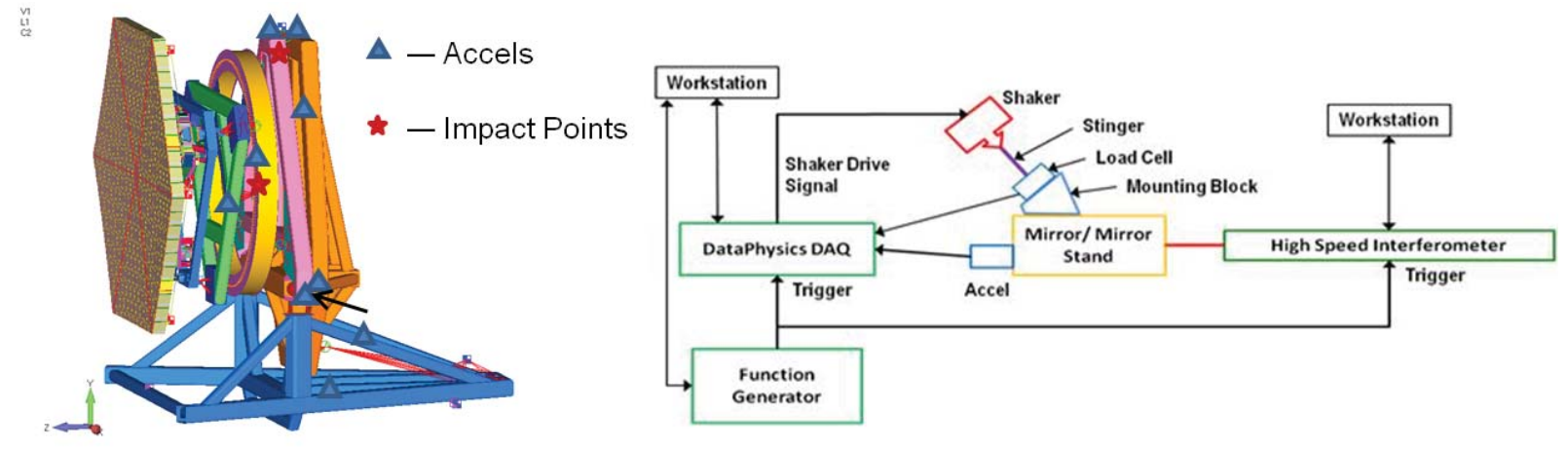

Figure 9. a) Test Configuration and Model. b) Test Configuration Data and Control Signal Flow.

Dynamic measurement data processing and analysis required development of custom software. A typical measurement involves a substantial amount of data. A typical single data set acquired at $900 \mathrm{~Hz}$ for 10 seconds resulting in 9000 exposures. The software is an extension of software used in a previous metrology project on $\mathrm{JWST}^{8}$. Dynamics measurements/calculations are performed at the independent individual pixel level. Phase unwrapping is performed in time domain per pixel and a PSD spectrum of selected parameters are calculated per pixel. The surface profile is a value of all the pixels at a given time. Modes are changes in surfaces in the measurement time interval. Both random and sine vibration are used to stimulate the mirror. Treating each pixel individually and temporal phase unwrapping each pixel results in Power Spectral Densities (PSD) per pixel. These PSDs were verified by comparing them to PSDs from accelerometers. At each specific time we can combine all pixels to get the surface profile. Zernike polynomials are then fit to each surface and each Zernike coefficients plotted in time. Fourier Transform gives a spectrum of that Zernike coefficient. Measuring transfer function of each segment leads to transfer function of the whole primary mirror. This enables extraction of backplane contribution to the behavior of individual segments. Interferometer data is normalized to input signal and displacement to force function (nm per Newton). Phase Delay measurements can be done by looking at the phase portion of the transfer functions at each given frequency. 


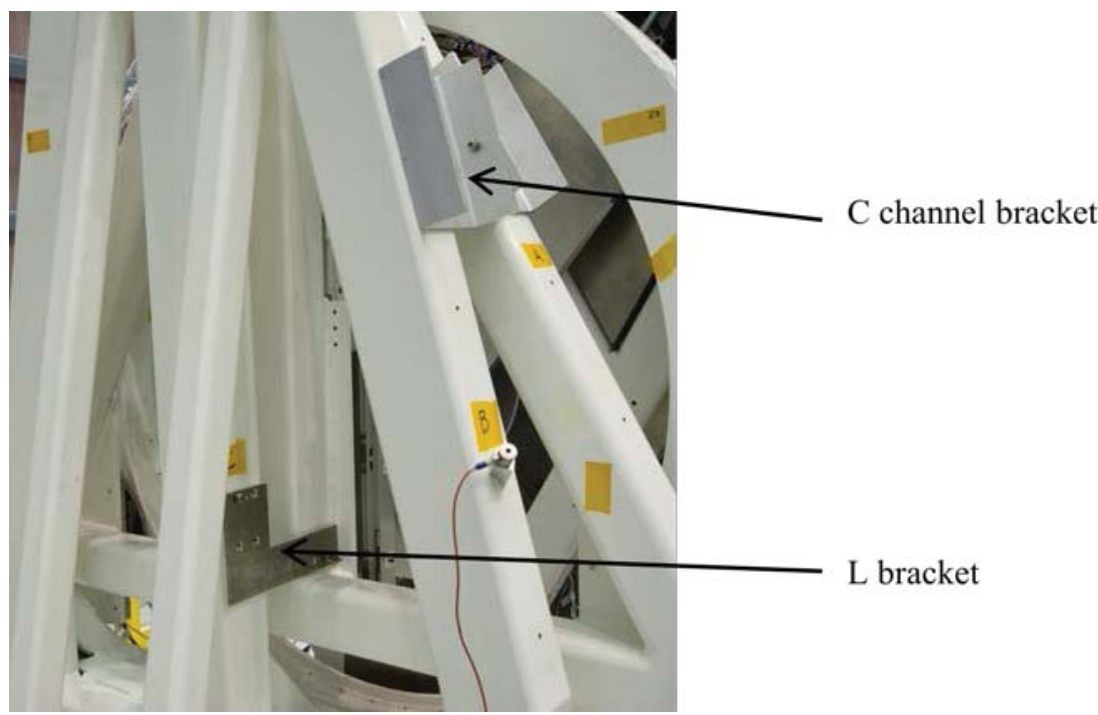

Figure 10. Structural Modifications made to the Test Stand.

During the course of the testing, various minor structural modification were made to the test stand to simulate both subtle and not so subtle ways that the real test article (OTIS) might change as a result of the acoustic test environment. Figure 10 shows two aluminum brackets used to effect the structural changes by changing the load path through the test stand. The upper right bracket, referred to as the $\mathrm{C}$ channel bracket changed stiffness and frequency as well as phase transfer function and gain transfer function (MTF). The changes were fairly significant (frequency shifts of $2 \%-5 \%$ ), while the lower left bracket, referred to as the L bracket, caused very subtle changes, often only discernible in the phase of the transfer function. The brackets generally were only used individually rather than together, as shown in the figure. In addition, mass was added directly to the mirror segment itself in the form of a plate that was fastened to one of the six hexagonal edges of the mirror. Two plates were made; one out of steel and one out of aluminum, which gave a fairly wide range of mass changes to the mirror of $6 \mathrm{~kg}$ and $2 \mathrm{~kg}$, respectively, of additional mass. Figure 11 shows the sine vibe PDS astigmatism for the different weights added to the mirror attached to one of the six hexagonal sides of the mirror to simulate a change.. 


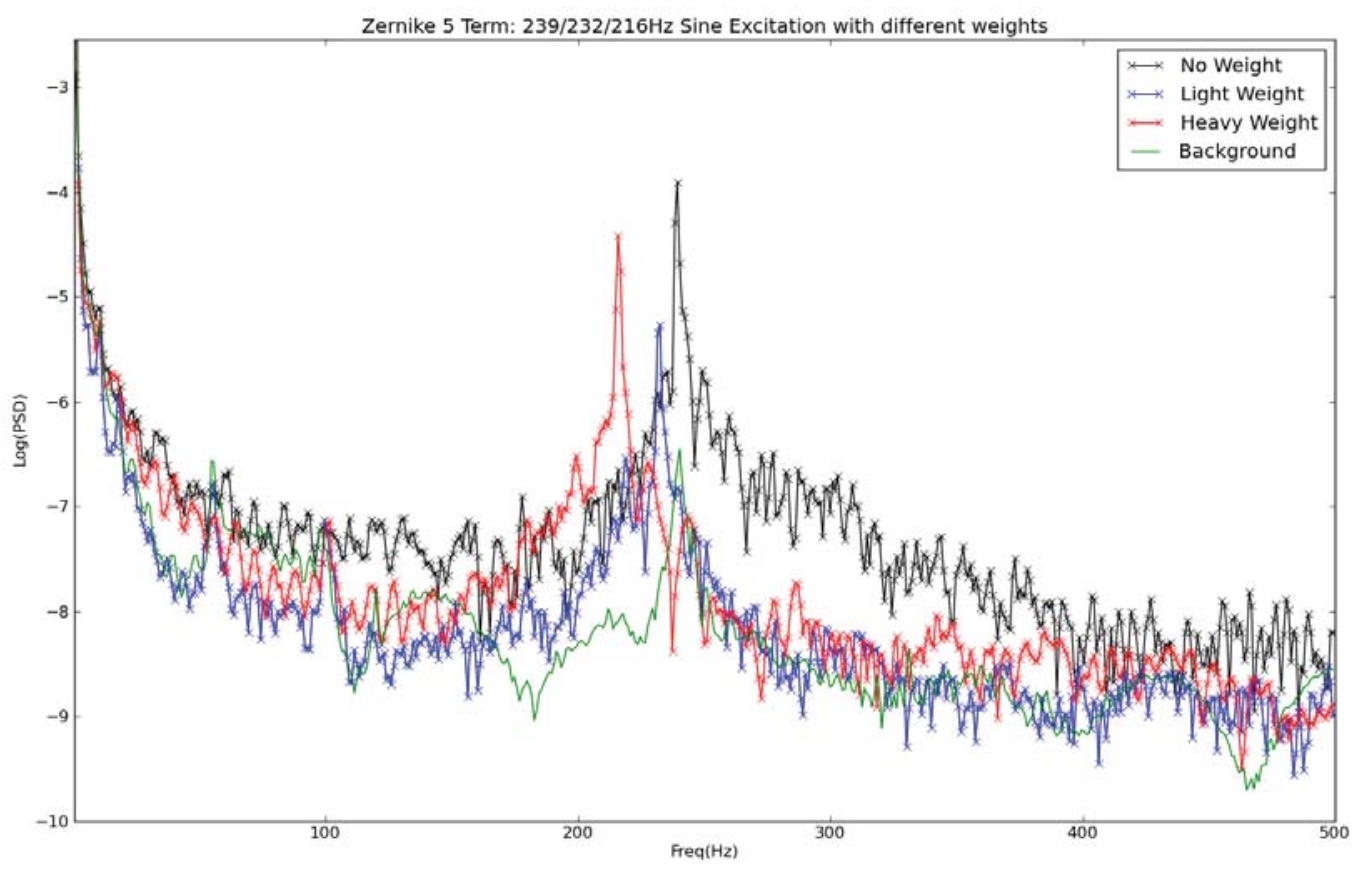

Figure 11. Sine Vibe PSD astigmatism, all pixels, for different weights added to the mirror attached to one of the six hexagonal sides of the mirror to simulate a change.

A large number of tests were conducted using both random and sine dwell excitation to map the response of the mirror at resonant frequencies and to statistically evaluate repeatability of the data. During each testing session, background measurements were frequently conducted. Although random excitation was necessary to identify resonant frequencies, sine dwell was the excitation of choice, as the SNR of sine dwell was quite high $(>25 \mathrm{~dB})$ compared to the SNR of random excitation $(\sim 8-9 \mathrm{~dB})$. In spite of the relatively low SNR during random excitation, the quality of the optical data was excellent, although the statistical repeatability was lower. Testing was also conducted during various times of the day and night to evaluate different background noise levels.

Figures 12 and 13 show the gain and phase lag transfer functions at $237 \mathrm{~Hz}$. Figure 12 is the baseline. Figure 13 shows the transfer functions after adding the $\mathrm{C}$-channel bracket shown in figure 10 on the test stand.

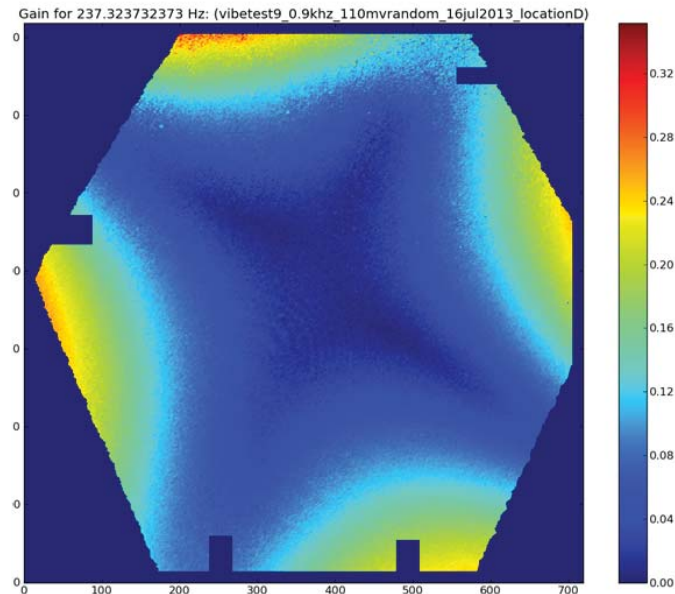

Gain at $237 \mathrm{~Hz}$ Baseline

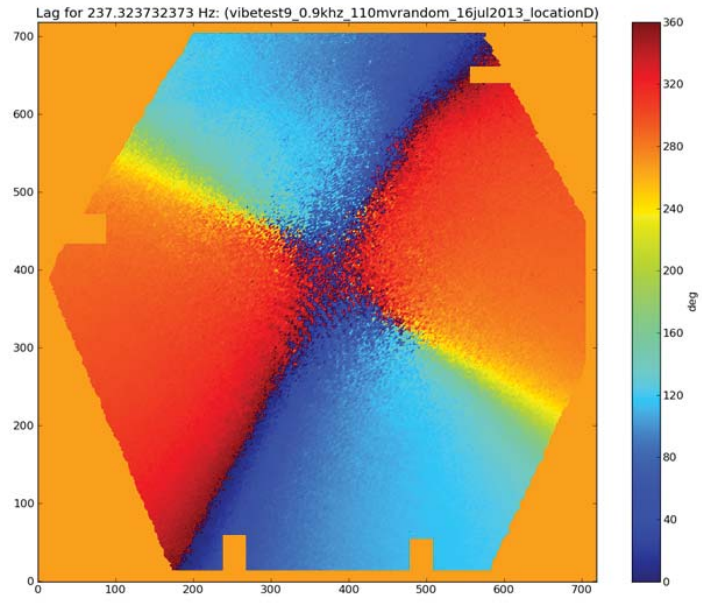

Phase Lag at $237 \mathrm{~Hz}$ Baseline 
Figure 12. Baseline Gain and phase lag at $237 \mathrm{~Hz}$.

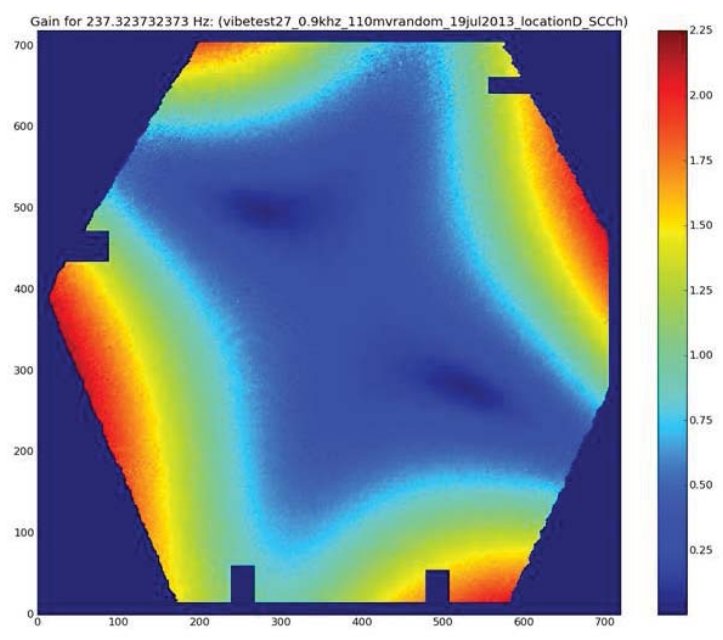

Gain at $237 \mathrm{~Hz}$ C-Channel Added

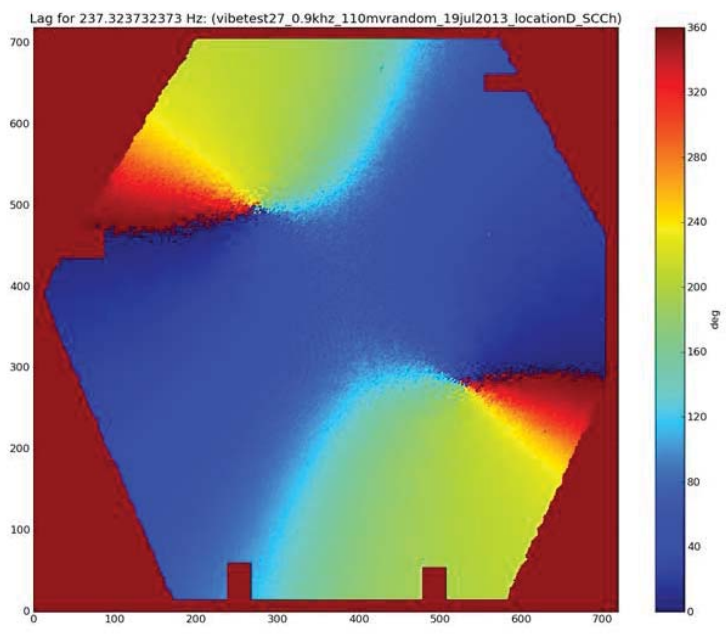

Phase Lag at $237 \mathrm{~Hz}$ C-Channel Added

Figure 13. Gain and phase lag at $237 \mathrm{~Hz}$ with the C-channel bracket installed on the test stand.

Gain pattern differences between structural configurations are subtle in pattern, but large in magnitude. The dominant astigmatic content is evident in the pattern. Phase lag pattern shows a clocking shift of about $90^{\circ}$ equates to $\sim 1$ milli-second shift in time of flight.

\section{SUMMARY}

We have established a capability to take accurate and repeatable PMSA surface figure measurements in the SSDIF. Static type workmanship testing at the PMSA level is possible and enabled by improved temperature stability in the SSDIF. A High speed interferometer (HSI) was developed to allow dynamic type testing of PMSAs for workmanship. HSI dynamic surface measurements were obtained successfully, demonstrating the capability to observe structural changes in the structure holding the PMSA. Dynamics testing will be used for diagnostics of the structural conditions of the OTIS and the measurements may help build confidence in telescope and OTIS dynamics models. Data processing is a challenge due to the large volumes of data. This requires large amounts of storage space and computing power. We are developing diagnostics criteria to determine the level of significance of any noted changes between before and after vibe and acoustics.

\section{REFERENCES}

1. Sabelhaus, P., Decker, J., “An Overview of the James Webb Space Telescope (JWST) Project”, Proceedings of SPIE, Vol. 5487, p 550-563 (2004).

2. Atkinson, C., Texter, S., Hellekson, R., Patton, K., Keski-Kuha, R., Feinberg, L., "Status of the JWST Optical Teles, cope Element”, Proceedings of SPIE, Vol. 6265, 62650T (2006).

3. Feinberg, L.D, Clampin, M., Keski-Kuha, R., Atkinson, C., Texter, S. "James Webb Space Telescope Optical Element Development History and Results", Proc. SPIE 8442, (2012).

4. Atkinson, C, Texter, S., Keski-Kuha R., Feinberg L., "Status of the JWST Optical Telescope Element", Proc SPIE 8442, (2012).

5. 4D Technology, in Tucson, Arizona, 4dtechnology.com. 
6. Hadaway, J. B, Chaney, D.M, Carey, L. M, "The optical metrology system for cryogenic testing of the JWST primary mirror segments", Proc. SPIE 8126, Optical Manufacturing and Testing IX, 81260P (September 26, 2011).

7. Chaney, D. M, Hadaway, J. B, Lewis, J., Gallagher, B., Brown, B., "Cryogenic performance of the JWST primary mirror segment engineering development unit", Proc. SPIE. 8150, Cryogenic Optical Systems and Instruments XIII, 815008. (September 08, 2011).

8. B. Saif, M. Bluth, P. Greenfield, W. Hack, B. Eegholm, P. Blake, R. Keski-Kuha, L. Feinberg, and J. W. Arenberg, "Measurement of large cryogenic structures using a spatially phase-shifted digital speckle pattern interferometer", Applied Optics, Vol. 47, Issue 6, pp. 737-745 (2008). 